\title{
Integer Codes Correcting High-Density Byte Asymmetric Errors
}

\author{
Aleksandar Radonjic and Vladimir Vujicic
}

\begin{abstract}
In optical networks without optical amplifiers the number of received photons never exceeds the number of sent ones. Hence, upon transmission, only asymmetric $(1 \rightarrow 0)$ errors can occur. Motivated by this fact, in this letter we present a class of integer codes capable of correcting high-density asymmetric errors within a $b$-bit byte. Unlike classical codes, these codes use integer and lookup table operations. As a result, they can be implemented "for free", i.e. without modifying the network hardware.
\end{abstract}

Index Terms-Integer codes, error correction, asymmetric errors, look-up table.

\section{INTRODUCTION}

$\mathrm{E}$ RROR control codes are usually designed for use on binary symmetric channels, where the error probabilities $0 \rightarrow 1$ and $1 \rightarrow 0$ are equal. However, in certain systems, the errors have a highly asymmetric nature. For the purpose of this paper, the most interesting example are optical networks without optical amplifiers (ONWOAs) (e.g. local and access networks) [1]. In these networks, the number of received photons never exceeds the number of transmitted ones. Hence, upon transmission only asymmetric $(1 \rightarrow 0)$ errors can occur [2], [3]. Besides this, it is known that these errors affect small number of bits. More precisely, the experiments showed that $99 \%$ to $99.9 \%$ of all errors are $t$-bit errors $(1 \leq t \leq 4)$ confined to one or two adjacent bytes [4]-[6].

Another common feature of ONWOAs is high computing power of network nodes. This can be seen from the fact that exterior nodes (e.g. PCs and servers) contain general purpose processors, whereas interior nodes (e.g. switches and routers) are always equipped with network processors (NPs) [7], [8]. The only difference between these chips is that NPs integrate coprocessors for common kernels of computation (e.g. lookup and cryptographic operations). On the other hand, both these chips have integer execution units as well as the memory system including the caches [7], [8]. Hence, it can be said that they are designed for integer and lookup table operations.

Motivated by these facts, in this letter we present a new class of integer codes. The proposed codes, like those in [9][11], have several desirable properties including systematic structure, simple encoding/decoding procedures and fast error correction algorithm based on table lookups. However, unlike [9]-[11], the codes presented in this paper can correct two types of errors within a $b$-bit byte: single $t / d$ asymmetric errors

The authors are with the Institute of Technical Sciences of the Serbian Academy of Sciences and Arts, 11000 Belgrade, Serbia (e-mail: sasa_radonjic@yahoo.com; vujicicv@yahoo.com). and double adjacent $t^{\prime} / d$ asymmetric errors, where $1 \leq t^{\prime}<t<d$ and $p=b / d \geq 2$. Thanks to this feature, they are more suitable for use in ONWOAs than the codes proposed in [9]-[11].

The organization of this paper is as follows: Section 2 deals with the construction of integer codes capable of correcting single $t / d$ and double adjacent $t^{\prime} / d$ asymmetric errors in a $b$-bit byte (integer $\left(\mathrm{S}_{t / d} \mathrm{AEC}-\mathrm{DA}_{t^{\prime} / d} \mathrm{AEC}\right)_{b}$ codes) Section 3 explains the implementation strategy for these codes, while Section 4 concludes the letter. Table 1 shows the notations used in this work.

TABLE I

NOTATIONS USED IN THIS LETTER.

\begin{tabular}{||c|c||}
\hline Symbol & Meaning \\
\hline$B_{i}$ & Integer value of the $i$-th $b$-bit data byte at the sender side \\
\hline$C_{\mathrm{B}}$ & Integer value of the $b$-bit check-byte at the sender side \\
\hline$\hat{B}_{i}$ & Integer value of the received $i$-th $b$-bit data byte \\
\hline$\hat{C}_{\mathrm{B}}$ & Integer value of the received $b$-bit check-byte \\
\hline$C_{\hat{\mathrm{B}}}$ & Integer value of the $b$-bit check-byte at the receiver side \\
\hline
\end{tabular}

\section{CODES CONSTRUCTION}

\section{A. Encoding and Decoding Procedures}

Let $Z_{2^{b}-1}=\left\{0,1, \ldots, 2^{b}-2\right\}$ be the ring of integers modulo $2^{b}-1$, and let $C_{i}$ and $C_{k+1}$ be integers such that $C_{i} \in Z_{2^{b}-1} \backslash\{0,1\}$ and $C_{k+1}=-1$. Now, suppose that the data are divided into $k b$ bit bytes. In that case, the encoder will compute the checkbyte in the same way as in [9]-[11], i.e. by using the following operations:

$C_{\mathrm{B}}=\left[C_{1} \cdot B_{1}+\cdots+C_{k} \cdot B_{k}\right]\left(\bmod 2^{b}-1\right)=\sum_{i=1}^{k} C_{i} \cdot B_{i}\left(\bmod 2^{b}-1\right)$

At the receiver, the decoder will perform the same calculation

$C_{\hat{\mathrm{B}}}=\left[C_{1} \cdot \hat{B}_{1}+\cdots+C_{k} \cdot \hat{B}_{k}\right]\left(\bmod 2^{b}-1\right)=\sum_{i=1}^{k} C_{i} \cdot \hat{B}_{i}\left(\bmod 2^{b}-1\right)$

after which the syndrome $S$ will be formed

$S=\left[C_{\hat{\mathrm{B}}}-\hat{C}_{\mathrm{B}}\right]\left(\bmod 2^{b}-1\right)$

Obviously, when $S \neq 0$, the codeword is corrupted by one or more errors. Whether these errors can be corrected or not depends on the values of the coefficients $C_{i}$. On the other hand, the coefficient $C_{k+1}$ always has the same value, since it corresponds to the errors within the check-byte. 


\section{B. Necessary and Sufficient Conditions}

Definition 1. An error is called t/d asymmetric error if $t$ or fewer bits in a d-bit byte are in a $1 \rightarrow 0$ error, where $1 \leq t<d$. Definition 2. An error is called low-density byte asymmetric (LDBA) error if there exists one t/d asymmetric error within a $b$-bit byte, where $p=b / d \geq 2$.

Definition 3. An error is called high-density byte asymmetric (HDBA) error if there exists two adjacent $t^{\prime} / d$ asymmetric errors within a b-bit byte, where $1 \leq t^{\prime}<t<d$ and $p=b / d \geq 2$. To make these definitions more clear, we give examples of LDBA and HDBA errors (Fig. 1).

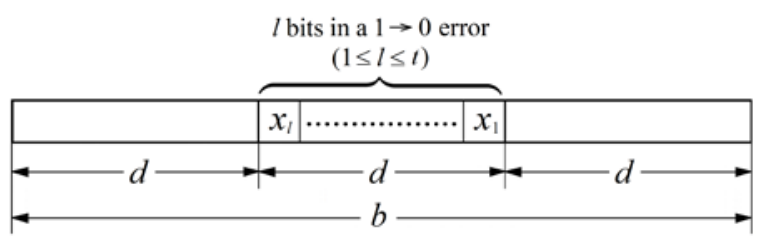

a)

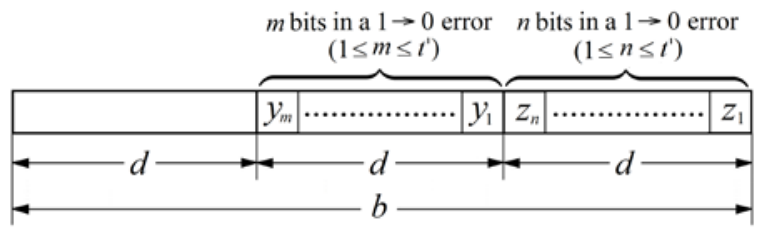

b)

Fig. 1. Examples of (a) LDBA errors and (b) HDBA errors, where $p=3$.

Definition 4. Let $0 \leq x_{1}<\cdots<x_{l}<d, 1 \leq l \leq t, 0 \leq r \leq p-1$, and let $e_{l, r}=\left\{\left(2^{x_{1}}+\cdots+2^{x_{1}}\right) \cdot 2^{d \cdot r}\right\}$ be the difference between the integer values of the correct $b$-bit byte and its received erroneous counterpart affected by LDBA error. Then, the set of syndromes corresponding to LDBA errors is defined as

$s_{1}=\left\{\bigcup_{i=1}^{k+1} \bigcup_{l=1}^{t} \bigcup_{r=0}^{p-1}\left(-C_{i} \cdot e_{l, r}\right)\left(\bmod 2^{b}-1\right)\right\}$

Definition 5. Let $0 \leq y_{1}<\cdots<y_{m}<d, 0 \leq z_{1}<\cdots<z_{n}<d$, $1 \leq m, n \leq t^{\prime}, 0 \leq s \leq p-2$ and let $e_{m, n, s}=\left\{\left(2^{y_{m}}+\cdots+2^{y_{1}}\right) \cdot 2^{d \cdot(s+1)}+\right.$ $\left.\left(2^{z_{n}}+\cdots+2^{z_{1}}\right) \cdot 2^{d \cdot s}\right\}$ be the difference between the integer values of the correct $b$-bit byte and its received erroneous counterpart affected by HDBA error. Then, the set of syndromes corresponding to HDBA errors is defined as

$S_{2}=\left\{\bigcup_{i=1}^{k+1} \bigcup_{m=1}^{t^{\prime}} \bigcup_{n=1}^{t^{\prime}} \bigcup_{s=0}^{p-2}\left(-C_{i} \cdot e_{m, n, s}\right)\left(\bmod 2^{b}-1\right)\right\}$

Now, we can prove the following theorem.

Theorem 1. The codes defined by (1)-(5) can correct all LDBA and HDBA errors iff there exist $k$ mutually different coefficients $C_{i} \in Z_{2^{b}-1} \backslash\{0,1\}$ such that

1. $\left|s_{1}\right|=(k+1) \cdot p \cdot \sum_{l=1}^{t}\left(\begin{array}{l}d \\ l\end{array}\right)$

2. $\left|s_{2}\right|=(k+1) \cdot(p-1) \cdot\left[\sum_{n=1}^{t^{\prime}}\left(\begin{array}{l}d \\ n\end{array}\right)\right]^{2}$

3. $s_{1} \cap s_{2}=\varnothing$

where $|\mathrm{A}|$ denotes the cardinality of $\mathrm{A}$, and $\mathrm{A} \cap \mathrm{B}$ the intersection of $\mathrm{A}$ and $\mathrm{B}$.
Proof. Condition 1 of this theorem says that LDBA errors generate $(k+1) \cdot p \cdot \sum_{l=1}^{t}\left(\begin{array}{l}d \\ l\end{array}\right)$ syndromes that are nonzero. To prove this, observe that the set $s_{1}$ can be expressed as

$S_{1}=\bigcup_{i=1}^{k+1} M_{i}$

where

$M_{1}=\left\{\bigcup_{l=1}^{t} \bigcup_{r=0}^{p-1}\left(-C_{1} \cdot e_{l, r}\right)\left(\bmod 2^{b}-1\right)\right\}$

$M_{k}=\left\{\bigcup_{l=1}^{t} \bigcup_{r=0}^{p-1}\left(-C_{k} \cdot e_{l, r}\right)\left(\bmod 2^{b}-1\right)\right\}$

$M_{k+1}=\left\{\bigcup_{l=1}^{t} \bigcup_{r=0}^{p-1}\left(e_{l, r}\right)\left(\bmod 2^{b}-1\right)\right\}$

Now, suppose that the coefficients $C_{i}$ are chosen in such a way that each one multiplied (modulo $2^{b}-1$ ) by each $e_{l, r}$ yields a different result. In that case, it will hold that

$M_{1} \cap \cdots \cap M_{k} \cap M_{k+1}=\varnothing$,

$\left|M_{1}\right|=\cdots=\left|M_{k}\right|=\left|M_{k+1}\right|$.

As a consequence, the set $s_{1}$ will have

$\left|s_{1}\right|=(k+1) \cdot\left|M_{k+1}\right|=(k+1) \cdot p \cdot \sum_{l=1}^{t}\left(\begin{array}{l}d \\ l\end{array}\right)$

nonzero elements. In a similar way Condition 2 says that HDBA errors generate $(k+1) \cdot(p-1) \cdot\left[\sum_{n=1}^{t^{\prime}}\left(\begin{array}{l}d \\ n\end{array}\right)\right]^{2}$ syndromes that are nonzero. To prove this, note that the set $s_{2}$ can be expressed as

$S_{2}=\bigcup_{i=1}^{k+1} N_{i}$

where

$N_{1}=\left\{\bigcup_{m=1}^{t^{\prime}} \bigcup_{n=1}^{t^{\prime}} \bigcup_{s=0}^{p-2}\left(-C_{1} \cdot e_{m, n, s}\right)\left(\bmod 2^{b}-1\right)\right\}$

$N_{k}=\left\{\bigcup_{m=1}^{t^{\prime}} \bigcup_{n=1}^{t^{\prime}} \bigcup_{s=0}^{p-2}\left(-C_{k} \cdot e_{m, n, s}\right)\left(\bmod 2^{b}-1\right)\right\}$

$N_{k+1}=\left\{\bigcup_{m=1}^{t^{\prime}} \bigcup_{n=1}^{t^{\prime}} \bigcup_{s=0}^{p-2}\left(e_{m, n, s}\right)\left(\bmod 2^{b}-1\right)\right\}$

Given this, suppose that the $C_{i}$ 's are chosen such that

$N_{1} \cap \cdots \cap N_{k} \cap N_{k+1}=\varnothing$

$\left|N_{1}\right|=\cdots=\left|N_{k}\right|=\left|N_{k+1}\right|$.

In that case, it is clear that the set $s_{2}$ will have

$\left|s_{2}\right|=(k+1) \cdot\left|N_{k+1}\right|=(k+1) \cdot(p-1) \cdot\left[\sum_{n=1}^{t^{\prime}}\left(\begin{array}{l}d \\ n\end{array}\right)\right]^{2}$

nonzero elements.Finally, Condition 3 is a necessary condition for distinguishing LDBA errors from HDBA errors. Therefore, the codes that satisfy the above conditions are $(k b+b, k b)$ integer $\left(\mathrm{S}_{t / d} \mathrm{AEC}-\mathrm{DA}_{\mathrm{t}^{\prime} / d} \mathrm{AEC}\right)_{b}$ codes. $\square$ 
Theorem 2. Let $\xi$ be the error set for $(k b+b, k b)$ integer $\left(S_{t / d} A E C-D A_{t^{\prime} / d} A E C\right)_{b}$ codes. Then,

$$
|\xi|=\left|s_{1}\right|+\left|s_{2}\right|=(k+1) \cdot\left[p \cdot \sum_{l=1}^{t}\left(\begin{array}{l}
d \\
l
\end{array}\right)+(p-1) \cdot\left[\sum_{n=1}^{t^{\prime}}\left(\begin{array}{l}
d \\
n
\end{array}\right)\right]^{2}\right] \text {. }
$$

Proof. This theorem follows from Theorem 1. $\square$

From Theorems 1 and 2 it is easy to see that the elements of $\xi$ cannot be generated without using a computer. Thus, it is clear that for some particular values of $t^{\prime}, t, d$ and $b$ we cannot know a priori the number and the values of coefficients $C_{i}$. In this letter, we have restricted ourselves to practical codes, i.e. to codes with parameters $t^{\prime}=3, t=4, d=8, b=32$ and $k \leq 64$. The results of the corresponding computer search are given in Table 2.

TABLE II

FIRST 64 COEFFICIENTS FOR INTEGER $\left(\mathrm{S}_{4 / 8} \mathrm{AEC}-\mathrm{DA}_{3 / 8} \mathrm{AEC}\right)_{32}$ CODES.

\begin{tabular}{||c|c|c|c|c|c|c|c||}
\hline 2 & 127 & 255 & 511 & 767 & 967 & 1007 & 1019 \\
\hline 1087 & 1151 & 1279 & 1567 & 1663 & 1727 & 1747 & 1927 \\
\hline 1999 & 2011 & 2029 & 2047 & 2447 & 2503 & 2539 & 2549 \\
\hline 2557 & 2591 & 2623 & 2687 & 2741 & 2813 & 2879 & 2887 \\
\hline 3023 & 3061 & 3063 & 3067 & 3071 & 3229 & 3253 & 3257 \\
\hline 3271 & 3301 & 3359 & 3527 & 3529 & 3571 & 3581 & 3583 \\
\hline 3623 & 3631 & 3733 & 3834 & 3847 & 3851 & 3853 & 4007 \\
\hline 4019 & 4073 & 4091 & 4159 & 4222 & 4247 & 4479 & 4567 \\
\hline \hline
\end{tabular}

\section{Error Correction Procedure}

From Theorem 2 we know that LDBA and HDBA errors generate $|\xi|$ nonzero syndromes. In addition, from the same theorem, we implicitly know that the relationship between the nonzero syndrome (element of the set $\xi$ ), error location (i) and error vector (e) can be described using (4)-(5). Both these facts imply that the syndrome table requires $|\xi| \times\left[2 \cdot b+\left\lceil\log _{2}(k+1)\right\rceil\right]$ bits (Fig. 2) to store the error correction data.

\begin{tabular}{|l|l|l|}
\hline Element of the set $\xi$ & Error location $(i)$ & Error vector $(e)$ \\
\hline & & \\
\hline
\end{tabular}

Fig. 2. Bit-width of one syndrome table entry.

Given this, suppose that that the data are received in error $(S \neq 0)$. In that case, the decoder will first search the syndrome table to find the appropriate entry. After that, it will execute one of the following operations:

- for LDBA errors within the $i$-th data byte

$B_{i}=\left[\hat{B}_{i}+e\right]\left(\bmod 2^{b}-1\right), 1 \leq i \leq k$;

$e=\left[e_{l, r}\right]\left(\bmod 2^{b}-1\right), 1 \leq l \leq t, 0 \leq r \leq p-1$;

- for LDBA errors within the check-byte

$C_{\mathrm{B}}=\left[\hat{C}_{\mathrm{B}}+e\right]\left(\bmod 2^{b}-1\right)$;

$e=\left[e_{l, r}\right]\left(\bmod 2^{b}-1\right), 1 \leq l \leq t, 0 \leq r \leq p-1$;

- for HDBA errors within the $i$-th data byte

$B_{i}=\left[\hat{B}_{i}+e\right]\left(\bmod 2^{b}-1\right), 1 \leq i \leq k$;

$e=\left[e_{m, n, s}\right]\left(\bmod 2^{b}-1\right), 1 \leq m, n \leq t^{\prime}, 0 \leq s \leq p-2$;

- for HDBA errors within the $i$-th check-byte

$C_{\mathrm{B}}=\left[\hat{C}_{\mathrm{B}}+e\right]\left(\bmod 2^{b}-1\right)$;

$e=\left[e_{m, n, s}\right]\left(\bmod 2^{b}-1\right), 1 \leq m, n \leq t^{\prime}, 0 \leq s \leq p-2$;
From the above it is clear that the efficiency of the error correction procedure (in terms of processing time) depends on the number of table lookups. For this reason it is desirable that the elements of $\xi$ are sorted in increasing order. In that case it is possible to use binary search algorithm, which requires $n_{\mathrm{TL}}$ table lookups $\left(1 \leq n_{\mathrm{TL}} \leq\left\lfloor\log _{2}|\xi|\right\rfloor+2\right)[12]$.

Example 1. Let $b=8, d=4, t=3, t^{\prime}=1, k=1$ and $C_{1}=2$. According to Theorem 2, the syndrome table will have $|\xi|=88$ entries. Given this, let us assume that we want to transmit 8 bits of data, $\mathrm{D}=10110011$. In that case, after calculating the value of check-byte $C_{\mathrm{B}}$

$C_{\mathrm{B}}=\left[C_{1} \cdot B_{1}\right]\left(\bmod 2^{b}-1\right)=[2 \cdot 179](\bmod 255)=103$

the codeword $C_{\mathrm{W}}=1011001101100111$ will have 16 bits. Now, let us analyze the following scenarios.

Scenario 1: Suppose that during data transmission an error on the $1^{\text {st }}, 3^{\text {rd }}$ and $4^{\text {th }}$ bit has occurred $\left(\hat{C}_{\mathrm{W}}=\underline{00000011}\right.$ 01100111). In that case, the decoder will calculate

$C_{\hat{\mathrm{B}}}=\left[C_{1} \cdot \hat{B}_{1}\right]\left(\bmod 2^{b}-1\right)=[2 \cdot 3](\bmod 255)=6$

$S=\left[C_{\hat{\mathrm{B}}}-\hat{C}_{\mathrm{B}}\right]\left(\bmod 2^{b}-1\right)=[6-103](\bmod 255)=158$

in order to check whether the value $S=158$ belongs to the set $\xi$ (Table 3). After completing this task, it will perform error correction by using

$B_{1}=\left[\hat{B}_{1}+e\right]\left(\bmod 2^{\mathrm{b}}-1\right)=[3+176](\bmod 255)=179$.

Scenario 2: Let us assume that during data transmission an error on the $10^{\text {th }}$ and $16^{\text {th }}$ bit has occurred $\left(\hat{C}_{\mathrm{W}}=10110011\right.$ 00100110). Similar to the previous case, after calculating

$C_{\hat{\mathrm{B}}}=\left[C_{1} \cdot \hat{B}_{1}\right]\left(\bmod 2^{b}-1\right)=[2 \cdot 179](\bmod 255)=103$

$S=\left[C_{\hat{\mathrm{B}}}-\hat{C}_{\mathrm{B}}\right]\left(\bmod 2^{b}-1\right)=[103-38](\bmod 255)=65$

the decoder will conclude that the value $S=65$ indicates an error within the check-byte (Table 3). As a consequence, the following procedure will take place:

$C_{\mathrm{B}}=\left[\hat{C}_{\mathrm{B}}+e\right]\left(\bmod 2^{b}-1\right)=[38+65](\bmod 255)=103$.

\section{IMPLEMENTATION STRATEGY}

From (1)-(3) and (6)-(9) it is clear that the encoder/decoder uses integer and lookup table (LUT) operations. Since these operations are supported by all processors, it is interesting to discuss how the proposed codes can be implemented on modern architectures. Without loss of generality, we will restrict ourselves to eight-core processors (Fig. 3) and integer $\left(\mathrm{S}_{4 / 8} \mathrm{AEC}-\mathrm{DA}_{3 / 8} \mathrm{AEC}\right)_{32}$ codes.

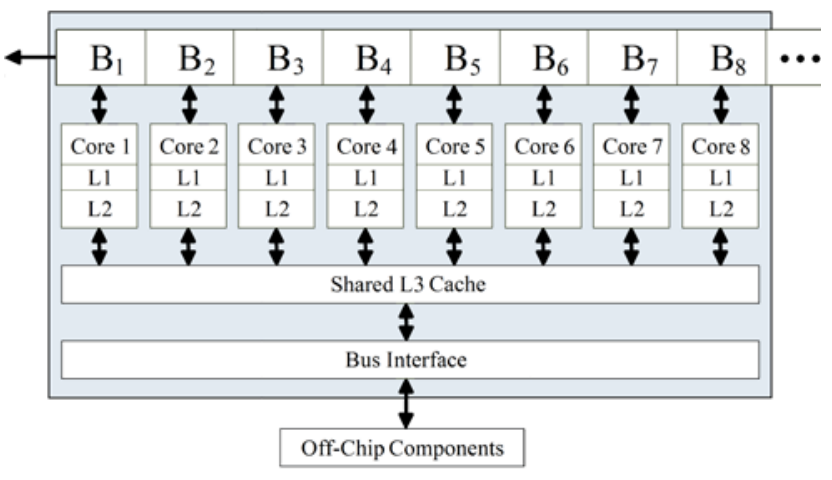

Fig. 3. Block diagram of eight-core processor. 
TABLE III

THE SYNDROME TABLE $\left(\operatorname{LUT}_{2}\right)$ FOR $(16,8)$ INTEGER $\left(\mathrm{S}_{3 / 4} \mathrm{AEC}-\mathrm{DA}_{1 / 4} \mathrm{AEC}\right)_{8}$ DECODER.

\begin{tabular}{|c|c|c|c|c|c|c|c|c|c|c|c|c|c|c|c|}
\hline & $\begin{array}{c}\text { Element } \\
\text { of the set } \xi\end{array}$ & $i$ & $e$ & & $\begin{array}{c}\text { Element } \\
\text { of the set } \xi\end{array}$ & $i$ & $e$ & & $\begin{array}{c}\text { Element } \\
\text { of the set } \xi\end{array}$ & $i$ & $e$ & & $\begin{array}{c}\text { Element } \\
\text { of the set } \xi\end{array}$ & $i$ & $e$ \\
\hline 1 & 1 & 2 & 1 & 23 & 34 & 2 & 34 & 45 & 128 & 2 & 128 & 67 & 222 & 1 & 144 \\
\hline 2 & 2 & 2 & 2 & 24 & 36 & 2 & 36 & 46 & 129 & 2 & 129 & 68 & 223 & 1 & 16 \\
\hline 3 & 3 & 2 & 3 & 25 & 40 & 2 & 40 & 47 & 130 & 2 & 130 & 69 & 224 & 2 & 224 \\
\hline 4 & 4 & 2 & 4 & 26 & 48 & 2 & 48 & 48 & 132 & 2 & 132 & 70 & 227 & 1 & 14 \\
\hline 5 & 5 & 2 & 5 & 27 & 62 & 1 & 224 & 49 & 136 & 2 & 136 & 71 & 229 & 1 & 13 \\
\hline 6 & 6 & 2 & 6 & 28 & 63 & 1 & 96 & 50 & 144 & 2 & 144 & 72 & 231 & 1 & 12 \\
\hline 7 & 7 & 2 & 7 & 29 & 64 & 2 & 64 & 51 & 158 & 1 & 176 & 73 & 233 & 1 & 11 \\
\hline 8 & 8 & 2 & 8 & 30 & 65 & 2 & 65 & 52 & 159 & 1 & 48 & 74 & 235 & 1 & 10 \\
\hline 9 & 9 & 2 & 9 & 31 & 66 & 2 & 66 & 53 & 160 & 2 & 160 & 75 & 237 & 1 & 9 \\
\hline 10 & 10 & 2 & 10 & 32 & 68 & 2 & 68 & 54 & 175 & 1 & 40 & 76 & 238 & 1 & 136 \\
\hline 11 & 11 & 2 & 11 & 33 & 72 & 2 & 72 & 55 & 176 & 2 & 176 & 77 & 239 & 1 & 8 \\
\hline 12 & 12 & 2 & 12 & 34 & 80 & 2 & 80 & 56 & 183 & 1 & 36 & 78 & 241 & 1 & 7 \\
\hline 13 & 13 & 2 & 13 & 35 & 94 & 1 & 208 & 57 & 187 & 1 & 34 & 79 & 243 & 1 & 6 \\
\hline 14 & 14 & 2 & 14 & 36 & 95 & 1 & 80 & 58 & 189 & 1 & 33 & 80 & 245 & 1 & 5 \\
\hline 15 & 16 & 2 & 16 & 37 & 96 & 2 & 96 & 59 & 190 & 1 & 160 & 81 & 246 & 1 & 132 \\
\hline 16 & 17 & 2 & 17 & 38 & 111 & 1 & 72 & 60 & 191 & 1 & 32 & 82 & 247 & 1 & 4 \\
\hline 17 & 18 & 2 & 18 & 39 & 112 & 2 & 112 & 61 & 192 & 2 & 192 & 83 & 249 & 1 & 3 \\
\hline 18 & 20 & 2 & 20 & 40 & 119 & 1 & 68 & 62 & 207 & 1 & 24 & 84 & 250 & 1 & 130 \\
\hline 19 & 24 & 2 & 24 & 41 & 123 & 1 & 66 & 63 & 208 & 2 & 208 & 85 & 251 & 1 & 2 \\
\hline 20 & 31 & 1 & 112 & 42 & 125 & 1 & 65 & 64 & 215 & 1 & 20 & 86 & 252 & 1 & 129 \\
\hline 21 & 32 & 2 & 32 & 43 & 126 & 1 & 192 & 65 & 219 & 1 & 18 & 87 & 253 & 1 & 1 \\
\hline 22 & 33 & 2 & 33 & 44 & 127 & 1 & 64 & 66 & 221 & 1 & 17 & 88 & 254 & 1 & 128 \\
\hline
\end{tabular}

1) $L 1$ caches. The $L 1$ cache is the smallest and fastest type of cache. Its size is limited to $64 \mathrm{~KB}$ (per core), whereas the data can be accessed in 1-5 clock cycles [8]. Both these facts suggest that the $\mathrm{L} 1$ can be used for storing the coefficient table $\left(\mathrm{LUT}_{1}\right.$ ) whose size is $k \times b$ bits (Table 4 ).

2) $L 2$ caches. The $L 2$ cache is somewhat slower and larger than the L1. Precisely, its size is limited to $512 \mathrm{~KB}$ (per core), whereas the individual entries can be accessed in 8-15 clock cycles [8]. Based on these parameters, it is clear that the L2 cannot be used for storing the syndrome table $\left(\mathrm{LUT}_{2}\right)$.

3) L3 cache. Unlike L1 and L2 caches, the L3 is shared among all cores. Due to this reason, this cache has the highest access latency (25-50 clock cycles). On the other hand, its size is sufficiently large (20-32 MB [8]) to store any $\mathrm{LUT}_{2}$.

4) Processing cores. From [7], [8] it is known that each core has at least one unit that performs 32/64-bit integer operations. Thus, it is clear that the encoding/decoding algorithm can be parallelized using eight threads. The similar applies for the error correction procedure. For instance, Core 1 can be used for performing binary search over first $|\xi| / 8$ entries, Core 2 for performing binary search over next $|\xi| / 8$ entries, and so on.

TABLE IV

LOOK-UP TABLE SizES FOR SOME INTEGER $\left(\mathrm{S}_{4 / 8} \mathrm{AEC} \mathrm{DA}_{3 / 8} \mathrm{AEC}\right)_{32}$ CODES.

\begin{tabular}{||c|c|c|c|c||}
\hline \multirow{2}{*}{ Code } & Encoder & \multicolumn{3}{|c||}{ Decoder } \\
\cline { 2 - 5 } & LUT $_{1}$ & LUT $_{1}$ & \multicolumn{2}{|c|}{ LUT $_{2}$} \\
\cline { 2 - 5 } & Size & Size & Size & $\begin{array}{c}\text { \# of Table } \\
\text { Lookups }\end{array}$ \\
\hline$(512,480)$ & $60 \mathrm{~B}$ & $60 \mathrm{~B}$ & $3.54 \mathrm{MB}$ & $1 \leq n_{\mathrm{TL}} \leq 20$ \\
\hline$(544,512)$ & $64 \mathrm{~B}$ & $64 \mathrm{~B}$ & $3.82 \mathrm{MB}$ & $1 \leq n_{\mathrm{TL}} \leq 20$ \\
\hline$(1024,992)$ & $124 \mathrm{~B}$ & $124 \mathrm{~B}$ & $7.19 \mathrm{MB}$ & $1 \leq n_{\mathrm{TL}} \leq 21$ \\
\hline$(1056,1024)$ & $128 \mathrm{~B}$ & $128 \mathrm{~B}$ & $7.52 \mathrm{MB}$ & $1 \leq n_{\mathrm{TL}} \leq 21$ \\
\hline$(2048,2016)$ & $252 \mathrm{~B}$ & $252 \mathrm{~B}$ & $14.58 \mathrm{MB}$ & $1 \leq n_{\mathrm{TL}} \leq 22$ \\
\hline$(2080,2048)$ & $256 \mathrm{~B}$ & $256 \mathrm{~B}$ & $15.02 \mathrm{MB}$ & $1 \leq n_{\mathrm{TL}} \leq 22$ \\
\hline
\end{tabular}

\section{CONCLUSION}

In this letter, we proposed a class of integer codes capable of correcting high-density asymmetric errors within a $b$-bit byte. We have shown that these codes can be implemented "for free", i.e. without modifying the network hardware. Thanks to this feature, the proposed codes have high potential to be used in practice. This primarily refers to optical networks without optical amplifiers in which all nodes possess powerful processors.

\section{REFERENCES}

[1] R. Ramaswani, K. Sivarajan and G. Sasaki, Optical Networks: A Practical Perspective, 3rd ed., Elsevier, Inc., 2010.

[2] J. R. Pierce, "Optical Channels: Practical Limits with Photon Counting," IEEE Trans. Communications, vol. 26, no.12, pp.1819-1821, Dec. 1978.

[3] P. Oprisan and B. Bose, "ARQ in Optical Networks," Proc. IEEE Int'l Symp. Pacific Rim Dependable Computing, pp. 251-257, Dec. 2001.

[4] CCITT Study Group XVIII Contribution D21, "Observations of Error Characteristics of Fiber Optic Transmission Systems,” Jan. 1989.

[5] D. Mello, E. Offer and J. Reichert, "Error Arrival Statistics for FEC Design in Four-Wave Mixing Limited Systems,"Proc. Optical Fiber Communication Conference, pp. 529-530, Mar. 2003.

[6] L. James, "Error Behaviour in Optical Networks", PhD thesis, Dept. of Engineering, University of Cambridge, 2005.

[7] R. Giladi, Network Processors: Architecture, Programming, and Implementation, Elsevier, Inc., 2008.

[8] L. Johnsson, "Introduction to HPC architecture," Dept. Computer Sciences, Univ. Houston, Houston, TX, USA, Jan. 2014.

[9] A. Radonjic and V. Vujicic, "Integer Codes Correcting Burst Errors within a Byte,” IEEE Trans. Computers, vol. 62, no. 2, pp. 411-415, Feb. 2013.

[10] A. Radonjic, K. Bala and V. Vujicic, "Integer Codes Correcting Double Asymmetric Errors," IET Communications, vol. 10, no. 14, pp. 16911696, Sep. 2016.

[11] A. Radonjic and V. Vujicic, "Integer Codes Correcting Spotty Byte Asymmetric Errors,” IEEE Comm. Letters, vol. 20, no. 12, pp. 23382341, Dec. 2016.

[12] K. Mehlhornand and P. Sanders, Algorithms and Data Structures: The Basic Toolbox, Springer, 2008. 